\title{
INEQUALITY CHAINS RELATED TO TRIGONOMETRIC AND HYPERBOLIC FUNCTIONS AND INVERSE TRIGONOMETRIC AND HYPERBOLIC FUNCTIONS
}

\section{ChAO-PING CHEN AND JÓZSEF SÁNDOR}

Abstract. We present inequality chains related to trigonometric and hyperbolic and inverse trigonometric and hyperbolic functions.

Mathematics subject classification (2010): 26D05, 26D07.

Keywords and phrases: Inequalities, trigonometric functions, hyperbolic functions, inverse trigonometric functions, inverse hyperbolic functions.

\section{REFERENCES}

[1] C.-P. ChEn AND J. SÁNDOR, Inequality chains for Wilker, Huygens and Lazarević type inequalities, available online at http://ajmaa.org/RGMIA/v15.php.

[2] I. LaZAREvić, Neke nejednakosti sa hiperbolickim funkcijama, Univerzitet u Beogradu. Publikacije Elektrotehničkog Fakulteta. Serija Matematika i Fizika, 170 1966, 41-48.

[3] B. J. MALEŠEvić, One method for proving inequalities by computer, J. Ineq. Appl. 2007, Article ID 78691.

[4] D. S. Mitrinović, Analytic Inequalities, Springer-Verlag, Berlin, 1970.

[5] E. NEUMAN, Inequalities involving inverse circular and inverse hyperbolic functions II, J. Math. Inequal. 4 (2010), 11-14.

[6] E. Neuman And J. SÁndor, On the Schwab-Borchardt mean II, Mathematica Pannonica 17 (2006), 49-59.

[7] E. Neuman And J. SÁNDOR, On some inequalities involving trigonometric and hyperbolic functions with emphasis on the Cusa-Huygens, Wilker, and Huygens inequalities, Math. Inequal. Appl. 13 (2010), 715-723.

[8] J. SÁNDOR, Trigonometric and Hyperbolic inequalities, available online at http://arxiv.org/abs /1105.0859.

[9] J. SÁNDor And M. Bencze, On Huygens' trigonometric inequality, RGMIA Res. Rep. Collection 8 (2005), no. 3, Article 14. 